\title{
QUANTIZATION NOISE REDUCTION IN OVERSAMPLED FILTER BANKS*
}

\author{
Helmut Bölcskei and Franz Hlawatsch \\ Institute of Communications and Radio-Frequency Engineering, Vienna University of Technology \\ Gusshausstrasse 25/389, A-1040 Vienna, Austria \\ phone: +43 158801 3527; fax: +43158705 83; e-mail: hboelcsk@aurora.nt.tuwien.ac.at
}

\begin{abstract}
We introduce a new method for quantization noise reduction in oversampled filter banks. This method is based on predictive quantization and achieves much better noise reduction than the best existing methods for noise reduction in overcomplete representations. It is demonstrated that the proposed oversampled predictive subband coders are well suited for subband coding applications where low resolution quantizers have to be used. In this case, oversampling combined with linear prediction improves the effective resolution of the subband coder at the cost of increased rate. Simulation results are provided to assess the achievable quantization noise reduction and resolution enhancement.
\end{abstract}

\section{INTRODUCTION AND OUTLINE}

Recently, oversampled filter banks (FBs) [1]-[7] have received increased attention, which is mainly due to their noise reducing properties and increased design freedom. In this paper, we introduce a new technique for quantization noise reduction in oversampled FBs. This technique is based on predictive quantization. The corresponding oversampled subband coders can be viewed as extensions of oversampled predictive A/D converters $[8,9]$ and of critically sampled predictive subband coders [10]-[12]. Our coder exploits two types of redundancies: the natural redundancy inherent in the input signal and the synthetic redundancy introduced by the oversampled analysis FB. The latter redundancy has previously been exploited for noise reduction by means of noise shaping $[6,7,13]$.

We show that predictive quantization in oversampled FBs yields better noise reduction (at the cost of increased bit rate) than the best methods previously proposed for noise reduction in overcomplete representations [14]-[16]. Oversampled predictive subband coders allow to trade bit rate for quantizer accuracy, and they are therefore well suited for subband coding applications where for technological or other reasons quantizers with low accuracy (even single bit) have to be used. The practical advantages of using low-resolution quantizers at the cost of increased rate are indicated by the popular sigma-delta techniques $[8,9]$. Using low-resolution quantizers in the digital domain increases circuit speed and reduces circuit complexity. One-bit codewords, for example, eliminate the need for word-framing [10].

This paper is organized as follows. Section 2 introduces the new subband coder. In Section 3 , we show how the optimum MIMO prediction system can be calculated. Section 4 presents examples demonstrating the benefit of oversampling and interchannel prediction. Finally, simulation results are provided in Section 5 .

${ }^{*}$ Funding by FWF grants P10531-ÖPY and P12228-TEC.

\section{OVERSAMPLED PREDICTIVE SUBBAND CODERS}

A block diagram of the proposed oversampled predictive subband coder is shown in Fig. 1. A major difference from oversampled predictive $A / D$ converters $[8,9]$ is that here we have to deal with a vector prediction problem, i.e., the predictor is a MIMO system.

The coder uses an oversampled, uniform FB $[17,18,4,2]$ with $N$ channels (subbands), subsampling factor $M<N$, analysis filters $h_{k}[n] \leftrightarrow H_{k}(z)$, and synthesis filters $f_{k}[n] \leftrightarrow$ $F_{k}(z)(k=0,1, \ldots, N-1)$. The analysis FB is represented by the $N \times M$ analysis polyphase matrix $[17,18] \mathbf{E}(z)$ defined as $[\mathbf{E}(z)]_{k, n}=\sum_{m=-\infty}^{\infty} h_{k}[m M-n] z^{-m}$. Similarly, the synthesis FB is represented by the $M \times N$ synthesis polyphase matrix $\mathbf{R}(z)$ defined as $[\mathbf{R}(z)]_{n, k}=\sum_{m=-\infty}^{\infty} f_{k}[m M+n] z^{-m}$. The analysis FB (see Fig. 1(a)) produces the $N \times 1$ vector of subband signals $\mathrm{v}[m]$ with $z$-transform

$$
\mathbf{v}(z)=\sum_{m=-\infty}^{\infty} \mathbf{v}[m] z^{-m}=\mathbf{E}(z) \mathbf{x}(z)
$$

where $\mathbf{x}(z)=\left[X_{0}(z) X_{1}(z) \ldots X_{M-1}(z)\right]^{T}$ with the polyphase components $X_{n}(z)=\sum_{m=-\infty}^{\infty} x[m M+n] z^{-m}$. The quantizer (labeled ' $Q$ ' in Fig. 1(a)) is modeled as additive noise $\mathbf{q}[m]$ with $z$-transform $\mathbf{q}(z)=\sum_{m=-\infty}^{\infty} \mathbf{q}[m] z^{-m}$.

The linear predictor uses the past quantized (i.e., noise-

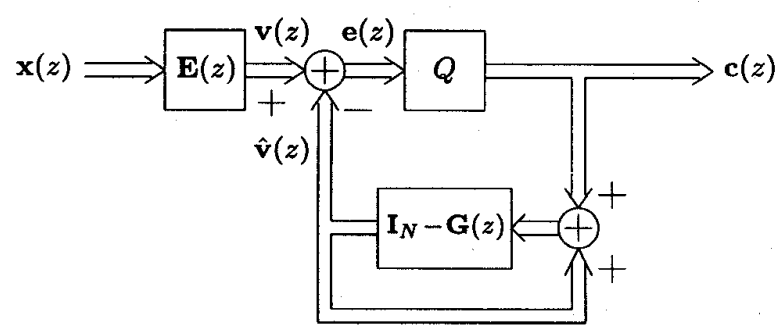

(a)

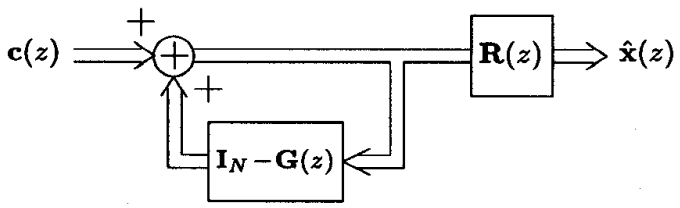

(b)

Fig. 1. Oversampled predictive subband coder: (a) encoder, (b) decoder. 
contaminated) subband signal vectors $\mathrm{v}[m-l]+\mathrm{q}[m-l]$ $(l=1,2, \ldots, L)$ to produce an estimate $\hat{v}[m]$ of the current subband signal vector $\mathbf{v}[m]$,

$$
\hat{\mathbf{v}}[m]=\sum_{l=1}^{L} \mathbf{G}_{l}[\mathbf{v}[m-l]+\mathbf{q}[m-l]],
$$

with the $L$ predictor coefficient matrices $\mathbf{G}_{l}$ of size $N \times N$. The predictor is a strictly causal $N \times N$ MIMO system of order $L$, with transfer function matrix

$$
\mathbf{I}_{N}-\mathbf{G}(z) \triangleq \sum_{l=1}^{L} \mathbf{G}_{l} z^{-l}
$$

Here, $\mathbf{I}_{N}$ denotes the $N \times N$ identity matrix, and $\mathbf{G}(z)=$ $\mathbf{I}_{N}-\sum_{l=1}^{L} \mathbf{G}_{l} z^{-l}$ is the prediction error system. The predictor coefficient matrices $\mathbf{G}_{l}$ will in general not be diagonal, so that we are performing interchannel prediction in addition to intrachannel prediction. We note that a reduced-cost predictor may be obtained by restricting the interchannel prediction to a given number of adjacent channels (where interchannel correlations are expected to be strongest) [13].

The input to the quantizer is the prediction error $\mathrm{e}[\mathrm{m}]=$ $\mathbf{v}[m]-\hat{\mathbf{v}}[\mathrm{m}]$. Increasing the FB's oversampling factor $N / M$ causes more synthetic redundancy in the subband signals. This results in improved prediction accuracy, i.e., smaller prediction error $\mathrm{e}[\mathrm{m}]$ and thus smaller dynamic range of the quantizer input. From Fig. 1(a), the $z$-transform of $e[m]$ is

$$
\mathbf{e}(z)=\mathbf{G}(z) \mathbf{v}(z)-\left[\mathbf{I}_{N}-\mathbf{G}(z)\right] \mathbf{q}(z) .
$$

The decoder (see Fig. 1(b)) operates on the quantizer output $\mathbf{c}[m]=\mathbf{e}[m]+\mathbf{q}[m]$. It consists of the same predictor $\mathbf{I}_{N}-\mathbf{G}(z)$ described above and the synthesis FB $\mathbf{R}(z)$. The decoder's output is the reconstructed signal $\hat{\mathbf{x}}[n]$. Defining the polyphase vector $\hat{\mathbf{x}}(z)=\left[\begin{array}{lll}\hat{X}_{0}(z) & \hat{X}_{1}(z) \ldots \hat{X}_{M-1}(z)\end{array}\right]^{T}$ with $\hat{X}_{n}(z)=\sum_{m=-\infty}^{\infty} \hat{x}[m M+n] z^{-m}$ and using (1) and (4), we obtain the overall input-output relation

$$
\hat{\mathbf{x}}(z)=\mathbf{R}(z)[\mathbf{E}(z) \mathbf{x}(z)+\mathbf{q}(z)] .
$$

Assuming a FB with perfect reconstruction (PR), i.e., $\mathbf{R}(z) \mathbf{E}(z)=\mathbf{I}_{M}$, this simplifies to

$$
\hat{\mathbf{x}}(z)=\mathbf{x}(z)+\mathbf{R}(z) \mathbf{q}(z) .
$$

The reconstruction error, $\hat{\mathbf{x}}(z)-\mathbf{x}(z)=\mathbf{R}(z) \mathbf{q}(z)$, here equals the reconstruction error in a non-predictive subband coder using a PR FB. Thus, the use of prediction does not by itself yield improved accuracy. However, since the dynamic range of the quantizer input, $\mathrm{e}[m]=\mathbf{v}[m]-\hat{\mathbf{v}}[m]$, will be smaller than that of $\mathbf{v}[m]$ (the quantizer input in the absence of prediction), it is possible to improve the quantizer resolution for a fixed number of quantization intervals.

Since according to (2) the predictor uses the quantized past subband signals, we have to deal with a noisy vector prediction problem. In the case of high-resolution quantizers, the effect of quantization noise can be neglected, i.e., $\hat{\mathbf{v}}[m] \approx \sum_{l=1}^{L} \mathbf{G}_{l} \mathbf{v}[m-l]$. However, here we are primarily interested in the case of low-resolution quantization.

The MIMO system $\mathbf{G}(z)$ is said to be minimum phase or minimum delay if all the roots of $\operatorname{det} \mathbf{G}(z)=0$ lie inside the unit circle in the $z$-plane. This condition ensures that the inverse filter $\mathbf{G}^{-1}(z)$, and hence the feedback loop, will be stable [19]. In the noiseless case $(q(z)=0)$, it is shown in [19] that $\mathbf{G}(z)$ is minimum phase if the process $v[m]$ is stationary and nondeterministic. Although we were not able to prove the minimum phase property of $\mathbf{G}(z)$, we always observed stability of $\mathrm{G}^{-1}(z)$ in our simulation examples.

\section{OPTIMUM PREDICTION SYSTEM}

We now derive the optimum prediction system $\mathbf{I}_{N}-\mathbf{G}(z)$. The input signal $x[n]$ is assumed to be a real-valued, widesense stationary, zero-mean random process with autocorrelation function $C_{x}[l]=\mathcal{E}\{x[n] x[n-l]\} \quad(\mathcal{E}$ denotes the expectation operator). We consider the $\mathrm{FB}$ input vector $\mathbf{x}[m]=[x[m M] \quad x[m M+1] \ldots x[m M+M-1]]^{T}$ with correlation matrix function $\mathbf{C}_{x}[l]=\mathcal{E}\left\{\mathbf{x}[m] \mathbf{x}^{T}[m-l]\right\}$ and power spectral matrix $\mathbf{S}_{x}(z)=\sum_{l=-\infty}^{\infty} \mathbf{C}_{x}[l] z^{-l}$. We note that $\left[\mathbf{C}_{x}[l]\right]_{i, j}=C_{x}[l M+i-j](i, j=0,1, \ldots, M-1)$.

Assuming for simplicity a FB with real-valued filters, the $N \times N$ correlation matrix function of the subband signal process $\mathbf{v}[m]$ is obtained as

$$
\mathbf{C}_{v}[l]=\mathcal{E}\left\{\mathbf{v}[m] \mathbf{v}^{T}[m-l]\right\}=\sum_{i=-\infty}^{\infty} \mathbf{E}_{i} \sum_{j=-\infty}^{\infty} \mathbf{C}_{x}[j] \mathbf{E}_{i+j-l}^{T}
$$

where $\mathbf{E}_{l}$ is defined by $\mathbf{E}(z)=\sum_{l=-\infty}^{\infty} \mathbf{E}_{l} z^{-l}$. The power spectral matrix of $\mathbf{v}[m]$ is

$$
\mathbf{S}_{v}(z)=\sum_{l=-\infty}^{\infty} \mathbf{C}_{v}[l] z^{-l}=\mathbf{E}(z) \mathbf{S}_{x}(z) \tilde{\mathbf{E}}(z)
$$

where $\tilde{\mathbf{E}}(z)=\mathbf{E}^{H}\left(1 / z^{*}\right)$

The quantization noise $q[m]$ is assumed to be uncorrelated with $x[n]$, zero-mean, and wide-sense stationary with power spectral matrix $\mathbf{S}_{q}(z)$. With (4) and (1), the power spectral matrix of the prediction error $\mathbf{e}[m]$ is obtained as

$$
\begin{aligned}
\mathbf{S}_{e}(z)= & \mathbf{G}(z) \mathbf{S}_{v}(z) \tilde{\mathbf{G}}(z) \\
& +\left[\mathbf{I}_{N}-\mathbf{G}(z)\right] \mathbf{S}_{q}(z)\left[\mathbf{I}_{N}-\tilde{\mathbf{G}}(z)\right] .
\end{aligned}
$$

The optimum predictor minimizes the prediction error variance defined as

$$
\sigma_{e}^{2} \triangleq \frac{1}{N} \mathcal{E}\left\{\mathbf{e}^{H}[m] \mathbf{e}[m]\right\}=\frac{1}{N} \operatorname{Tr}\left\{\int_{-1 / 2}^{1 / 2} \mathbf{S}_{e}\left(e^{j 2 \pi \theta}\right) d \theta\right\},
$$

where $\operatorname{Tr}\{\cdot\}$ denotes the trace of a matrix. Inserting (3) and (6) into (7) yields after straightforward manipulations [7, 13]

$$
\begin{aligned}
\sigma_{e}^{2}=\frac{1}{N} \operatorname{Tr} & \left\{\mathbf{C}_{v}[0]-\sum_{l=1}^{L}\left[\mathbf{C}_{v}^{T}[l] \mathbf{G}_{l}+\mathbf{C}_{v}[l] \mathbf{G}_{l}^{T}\right]\right. \\
& \left.+\sum_{i=1}^{L} \mathbf{G}_{i} \sum_{l=1}^{L}\left[\mathbf{C}_{v}[l-i]+\mathbf{C}_{q}[l-i]\right] \mathbf{G}_{l}^{T}\right\}
\end{aligned}
$$

where we have used $\mathbf{C}_{v}^{T}[-l]=\mathbf{C}_{v}[l]$ and $\mathbf{C}_{q}^{T}[-l]=\mathbf{C}_{q}[l]$.

We shall next calculate the optimum predictor coefficient matrices $\mathbf{G}_{l}$. We assume real-valued $\mathbf{G}_{l}$ for simplicity. Setting $\frac{\partial \sigma_{e}^{2}}{\partial \mathbf{G}_{i}}=\mathbf{0}$ for $i=1,2, \ldots, L$, and using the matrix differentiation rules in [20], we obtain the following block Töplitz system of equations [7, 13],

$$
\sum_{l=1}^{L}\left[\mathbf{C}_{v}[l-i]+\mathbf{C}_{q}[l-i]\right] \mathbf{G}_{l}^{T}=\mathbf{C}_{v}^{T}[i], \quad i=1,2, \ldots, L .
$$

Using (9) in (8), the minimum prediction error variance is obtained as

$$
\sigma_{e, \min }^{2}=\frac{1}{N} \operatorname{Tr}\left\{\mathbf{C}_{v}[0]-\sum_{l=1}^{L} \mathbf{C}_{v}[l] \mathbf{G}_{l, \text { opt }}^{T}\right\}
$$

where $\mathbf{G}_{l, \mathrm{opt}}$ is the solution of (9). Note that the noise statistics are not explicitly contained in this expression, although they do influence $\sigma_{e, \min }^{2}$ via $\mathbf{G}_{l, \text { opt }}$. 
In the noiseless case, i.e., $\mathbf{C}_{q}[l] \equiv 0,(9)$ reduces to

$$
\sum_{l=1}^{L} \mathbf{C}_{v}[l-i] \mathbf{G}_{l}^{T}=\mathbf{C}_{v}^{T}[i], \quad i=1,2, \ldots, L .
$$

This can be solved efficiently using the multi-channel Levinson recursion [21]. Another important special case where this is possible is the noisy case with white (but possibly correlated) quantization noise, i.e., $\mathbf{C}_{q}[l]=\mathbf{C}_{q}[0] \delta[l]$. Here, (9) reduces to (11) with $\mathbf{C}_{v}[0]$ replaced by $\mathbf{C}_{v}[0]+\mathbf{C}_{q}[0]$. We finally note that the above derivation can easily be extended to incorporate correlations between $v[m]$ and $q[m]$.

\section{EXAMPLES}

As a simple example, let us consider a paraunitary twochannel FB (i.e., $N=2$ ) with $M=1$ and, hence, oversampling factor $N / M=2$. The analysis filters are the Haar filters $H_{0}(z)=\frac{1}{\sqrt{2}}\left(1+z^{-1}\right)$ and $H_{1}(z)=\frac{1}{\sqrt{2}}\left(1-z^{-1}\right)$, and the PR synthesis filters with minimum norm are $F_{0}(z)=\frac{1}{2} \tilde{H}_{0}(z)$ and $F_{1}(z)=\frac{1}{2} \tilde{H}_{1}(z)$. The input signal is modeled as an AR-1 process [22] defined by $x[n]=a x[n-1]+u[n]$ with $a=0.5$ and white driving noise $u[n]$ with variance 1 . With $C_{x}[l]=\frac{4}{3} 2^{-|l|}[22], \mathbf{E}_{0}=\frac{1}{\sqrt{2}}\left[\begin{array}{ll}1 & 1\end{array}\right]^{T}$, and $\mathbf{E}_{1}=\frac{1}{\sqrt{2}}\left[\begin{array}{ll}1 & -1\end{array}\right]^{T}$, (5) yields $\mathbf{C}_{v}[0]=\left[\begin{array}{cc}2 & 0 \\ 0 & 2 / 3\end{array}\right], \mathbf{C}_{v}[1]=\left[\begin{array}{cc}3 / 2 & 1 / 2 \\ -1 / 2 & -1 / 6\end{array}\right]$, $\mathbf{C}_{v}[-1]=\mathbf{C}_{v}^{T}[1]$, and $\mathbf{C}_{v}[l]=0$ otherwise.

In the absence of prediction (i.e., $L=0$ or $\mathbf{e}[m]=\mathbf{v}[m]$ ), the variance at the input of the quantizer is obtained as $\sigma_{e}^{2}=\sigma_{v}^{2}=4 / 3 \approx 1.33$. We shall now evaluate the variance reduction achieved by the optimum first-order (i.e., $L=1$ ) predictor $\mathbf{I}_{2}-\mathbf{G}_{\mathrm{opt}}(z)=\mathbf{G}_{1, \mathrm{opt}} z^{-1}$ in various situations.

No quantization noise. We first consider the case of no quantization noise (noiseless prediction). Here, it follows from (9) that the optimum coefficient matrix $\mathbf{G}_{1}$ is determined by $\mathbf{C}_{v}[0] \mathbf{G}_{1}^{T}=\mathbf{C}_{v}^{T}[1]$, which yields

$$
\mathbf{G}_{1, \mathrm{opt}}=\mathbf{C}_{v}[1] \mathbf{C}_{v}^{-1}[0]=\left[\begin{array}{cc}
3 / 4 & 3 / 4 \\
-1 / 4 & -1 / 4
\end{array}\right] \text {. }
$$

The corresponding minimum prediction error variance is obtained from (10) as $\sigma_{e, \min }^{2}=\frac{1}{2} \operatorname{Tr}\left\{\mathbf{C}_{v}[0]-\mathbf{C}_{v}[1] \mathbf{G}_{1, \text { opt }}^{T}\right\}=$ 0.5. (This should be compared with $\sigma_{e}^{2} \approx 1.33$.)

Uncorrelated, white quantization noise. We next assume uncorrelated, white quantization noise with variance 1 in each channel, i.e., $\mathbf{C}_{q}[l]=\mathbf{I}_{2} \delta[l]$. From (9),

$$
\mathbf{G}_{1, \mathrm{opt}}=\mathbf{C}_{v}[1]\left[\mathbf{C}_{v}[0]+\mathbf{I}_{2}\right]^{-T}=\left[\begin{array}{cc}
1 / 2 & 3 / 10 \\
-1 / 6 & -1 / 10
\end{array}\right] \text {. }
$$

The minimum prediction error variance is obtained from (10) as $\sigma_{e, \min }^{2}=\frac{1}{2} \operatorname{Tr}\left\{\mathbf{C}_{v}[0]-\mathbf{C}_{v}[1] \mathbf{G}_{1, \text { opt }}^{T}\right\}=5 / 6 \approx 0.83$, which is still better than $\sigma_{e}^{2} \approx 1.33$.

No interchannel prediction. Still assuming $\mathbf{C}_{q}[l]=$ $I_{2} \delta[l]$, let us compare the last result with that of a predictor without interchannel prediction. Replacing $\mathbf{G}_{1}$ with a diagonal matrix $\mathbf{G}_{1}^{D}=\operatorname{diag}\{\alpha, \beta\}$, it follows from (8) that $\sigma_{e}^{2}=\frac{4}{3}-\frac{3}{2} \alpha+\frac{1}{6} \beta+\frac{3}{2} \alpha^{2}+\frac{5}{6} \beta^{2}$. Setting $\frac{\partial \sigma_{e}^{2}}{\partial \alpha}=0$ and $\frac{\partial \sigma_{e}^{2}}{\partial \beta}=0$ yields $\alpha_{\mathrm{opt}}=1 / 2$ and $\beta_{\mathrm{opt}}=-1 / 10$, so that

$$
\mathbf{G}_{1, \mathrm{opt}}^{D}=\left[\begin{array}{cc}
1 / 2 & 0 \\
0 & -1 / 10
\end{array}\right] \text {. }
$$

Note that the diagonal elements of $\mathbf{G}_{1, \mathrm{opt}}^{D}$ equal those of $\mathbf{G}_{1, \mathrm{opt}}$ in (12). The prediction error variance is obtained as $\sigma_{e, \min }^{2}=0.95$. A comparison with the result obtained further above with interchannel prediction, $\sigma_{e, \min }^{2} \approx 0.83$, shows the benefit of exploiting interchannel correlation.

\section{SIMULATION RESULTS}

The performance of oversampled predictive subband coders will now be further analyzed using simulation results.

Exploiting synthetic redundancy. Our first simulation example demonstrates the predictor's ability to exploit the synthetic redundancy introduced by the oversampled analysis FB for improving prediction accuracy and hence for enhancing resolution.

We used a paraunitary, odd-stacked, cosine-modulated FB [23] with $N=16$ channels, normalized analysis filters (i.e. $\left\|h_{k}\right\|=1$ ) of length 64 , and various oversampling factors $K$. The input was white noise (no natural redundancy). Hence, all the prediction gain is due to the synthetic redundancy. In particular, this means that there will not be any prediction gain in the special case of a critically sampled paraunitary (orthogonal) FB (which does not introduce synthetic redundancy). For the sake of simplicity, we considered the case of no quantization (noiseless prediction).

Fig. 2(a) shows the theoretical minimum prediction error variance $10 \log \sigma_{e, \min }^{2}$, calculated according to (10), as

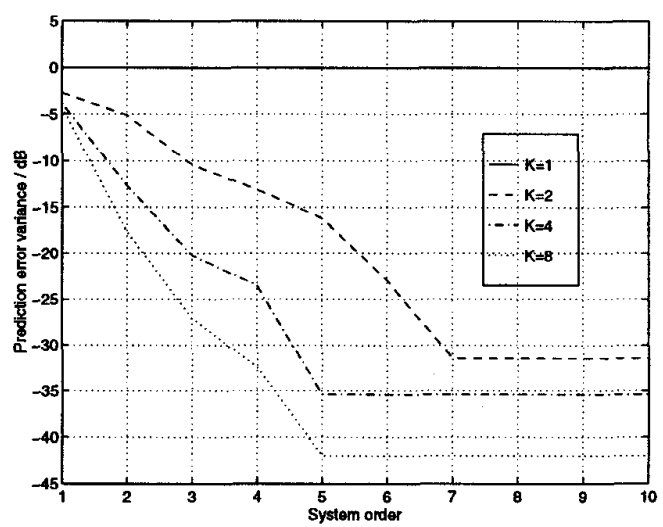

(a)

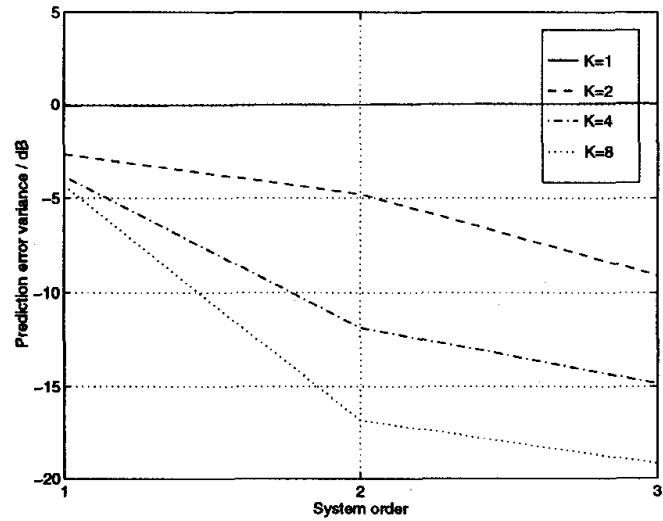

(b)

Fig. 2. Prediction error variance $10 \log \sigma_{e, \min }^{2}$ for a white noise input signal and no quantization noise as a function of the predictor order $L$ for various oversampling factors $K=N / M:$ (a) theoretical, (b) measured. 
a function of the predictor order $L$ for various oversampling factors $K=N / M$. For increasing $L, \sigma_{e, \min }^{2}$ is seen to decrease up to a certain point, after which it remains constant.

Fig. 2(b) shows the corresponding measured prediction error variance obtained for an implemented coder. This result was obtained by averaging over 5 realizations (of length 1024) of the input process. For predictor order $L>3$ (not shown), the performance of the implemented coder deteriorated significantly. This is probably due to the near-singularity of (9) for $L>3$, which introduces numerical errors in the computation of the prediction system. Note that for critical sampling $(K=1)$, there is in fact no prediction gain.

Improving effective quantizer resolution. Our next simulation example demonstrates that oversampling combined with linear prediction is a powerful means to improve the effective resolution of a subband coder. We coded realizations of an AR-1 process with length 1024 and correlation coefficient $a=0.5$ using a critically sampled, paraunitary, 16-channel, odd-stacked, cosine-modulated FB and quantizers with 152 quantization intervals (8-bit quantizers) in each channel. The resulting $\mathrm{SNR}=\frac{\|x\|^{2}}{\|\hat{x}-x\|^{2}}$ was $32.49 \mathrm{~dB}$. Next, we coded the same signal using an oversampled $\mathrm{FB}$ with oversampling factor $K=4$ and a predictor with order $L=10$ (designed under the assumption of uncorrelated and white quantization noise). Here, quantizers with only 15 quantization intervals (4-bit quantizers) achieved an SNR of $32.51 \mathrm{~dB}$. Hence, oversampling and prediction allowed us to save 4 bits of quantizer resolution in each of the 16 channels, of course at the cost of increased sample rate. For oversampling factor 8 , quantizers with 15 quantization intervals (4-bit quantizers), and a predictor with order $L=15$, we obtained an SNR of $50.48 \mathrm{~dB}$. In order to achieve an SNR of $50.43 \mathrm{~dB}$ with a critically sampled subband coder without prediction, we had to use 1219 quantization intervals (11-bit quantizers). Hence, oversampling and prediction here saved 7 bits of quantizer resolution. Table 1 summarizes these results.

\begin{tabular}{|r|r|r|r|}
\hline$K$ & $L$ & SNR/dB & $N_{Q}$ \\
\hline 1 & 0 & 32.49 & 152 \\
4 & 10 & 32.51 & 15 \\
1 & 0 & 50.43 & 1219 \\
8 & 15 & 50.48 & 15 \\
\hline
\end{tabular}

Table 1. Improving the effective resolution of a subband coder by means of oversampling and prediction. $\left(N_{Q}\right.$ denotes the number of quantization intervals required.)

\section{CONCLUSION}

We introduced a new method for quantization noise reduction in oversampled filter banks. This method is based on predictive quantization; it can be viewed as an extension of oversampled predictive A/D converters. We demonstrated that predictive quantization in oversampled filter banks yields considerable quantization noise reduction at the cost of increased rate. The combination of oversampled filter banks with linear prediction improves the effective resolution of subband coders and is thus well suited for applications where -for technological or other reasons-quantizers with low resolution (even single bit) have to be used. Using low resolution quantizers in the digital domain increases circuit speed and allows for lower circuit complexity.

\section{Acknowledgement}

The authors thank T. Stranz for writing the software used for producing the simulations.

\section{References}

[1] H. Bölcskei, F. Hlawatsch, and H. G. Feichtinger, "Frametheoretic analysis and design of oversampled filter banks," in Proc. IEEE ISCAS-96, vol. 2, (Atlanta (GA)), pp. 409-412, May 1996.

[2] H. Bölcskei, F. Hlawatsch, and H. G. Feichtinger, "Frametheoretic analysis of oversampled filter banks," IEEE Trans. Signal Processing, to appear 1998.

[3] Z. Cvetković, "Oversampled modulated filter banks and tight Gabor frames in $l^{2}(Z)$," in Proc. IEEE ICASSP-95, (Detroit (MI)), pp. 1456-1459, May 1995.

[4] Z. Cvetković and M. Vetterli, "Oversampled filter banks," IEEE Trans. Signal Processing, vol. 46, pp. 1245-1255, May 1998.

[5] H. Bölcskei, F. Hlawatsch, and H. G. Feichtinger, "Oversampled FIR and IIR DFT filter banks and Weyl-Heisenberg frames," in Proc. IEEE ICASSP-96, vol. 3, (Atlanta, GA), pp. 1391-1394, May 1996.

[6] H. Bölcskei and F. Hlawatsch, "Oversampled filter banks: Optimal noise shaping, design freedom, and noise analysis," in Proc. IEEE ICASSP-97, vol. 3, (Munich, Germany), pp. 2453-2456, April 1997.

[7] H. Bölcskei, Oversampled filter banks and predictive subband coders. PhD thesis, Vienna University of Technology, Nov. 1997.

[8] S. K. Tewksbury and R. W. Hallock, "Oversampled, linear predictive and noise-shaping coder of order $N>1$," IEEE Trans. Circuits and Systems, vol. 25, pp. 436-447, July 1978.

[9] J. C. Candy and G. C. Temes, Oversampling Delta-Sigma Data Converters. New York (NY): IEEE Press, 1993.

[10] N. S. Jayant and P. Noll, Digital Coding of Waveforms. Englewood Cliffs (NJ): Prentice Hall, 1984.

[11] P. W. Wong, "Rate distortion efficiency of subband coding with crossband prediction," IEEE Trans. Inf. Theory, vol. 43, pp. 352-356, Jan. 1997.

[12] L. Vandendorpe and B. Maison, "Multiple-input/multipleoutput prediction of subbands and image compression," in Proc. COST 254 (Emerging Techniques for Communication Terminals), (Toulouse, France), July 1997.

[13] H. Bölcskei and F. Hlawatsch, "Noise reduction in oversampled filter banks using predictive quantization," IEEE Trans. Inf. Theory, April 1998. submitted.

[14] Z. Cvetković and M. Vetterli, "Overcomplete expansions and robustness," in Proc. IEEE TFTS-96, (Paris, France), pp. 325-328, June 1996.

[15] V. K. Goyal, M. Vetterli, and N. T. Thao, "Quantized overcomplete expansions in $r^{N}$ : Analysis, synthesis and algorithms," IEEE Trans. Inf. Theory, vol. 44, pp. 16-31, Jan. 1998.

[16] N. J. Munch, "Noise reduction in tight Weyl-Heisenberg frames," IEEE Trans. Inf. Theory, vol. 38, pp. 608-616, March 1992.

[17] P. P. Vaidyanathan, Multirate Systems and Filter Banks. Englewood Cliffs (NJ): Prentice Hall, 1993.

[18] M. Vetterli and J. Kovačević, Wavelets and Subband Coding. Englewood Cliffs (NJ): Prentice Hall, 1995.

[19] A. Gersho and R. M. Gray, Vector Quantization and Signal Compression. Boston: Kluwer, 1992.

[20] A. Weinmann, Uncertain Models and Robust Control. Vienna, Austria: Springer, 1991.

[21] S. M. Kay, Modern Spectral Estimation. Englewood Cliffs (NJ): Prentice Hall, 1988

[22] A. Papoulis, Probability, Random Variables, and Stochastic Processes. New York: McGraw-Hill, 3rd ed., 1991.

[23] H. Bölcskei and F. Hlawatsch, "Oversampled cosine modulated filter banks with perfect reconstruction," IEEE Trans. Circuits and Systems II, Special Issue on Multirate Systems, Filter Banks, Wavelets, and Applications, vol. 45, Aug. 1998. 